\title{
Hemodialysis in MNGIE transiently reduces serum and urine levels of thymidine and deoxyuridine, but not CSF levels and neurological function
}

\author{
Benjamin Röeben ${ }^{1,2^{*}+}$, Justus Marquetand ${ }^{3 \dagger}$, Benjamin Bender ${ }^{4}$, Heiko Billing ${ }^{5}$, Tobias B. Haack ${ }^{6,7,8}$, \\ Iciar Sanchez-Albisua ${ }^{9}$, Ludger Schöls ${ }^{1,2}$, Henk J. Blom ${ }^{10}$ and Matthis Synofzik ${ }^{1,2}$
}

\begin{abstract}
Mitochondrial neurogastrointestinal encephalomyopathy (MNGIE) is a rare, autosomal-recessive mitochondrial disorder caused by TYMP mutations presenting with a multisystemic, often lethal syndrome of progressive leukoencephalopathy, ophthalmoparesis, demyelinating neuropathy, cachexia and gastrointestinal dysmotility. Hemodialysis (HMD) has been suggested as a treatment to reduce accumulation of thymidine and deoxyuridine. However, all studies so far have failed to measure the toxic metabolites in cerebrospinal fluid (CSF), which is the crucial compartment for CNS damage.

Our study is the first prospective, longitudinal investigation, exploiting detailed serial testing of predefined clinical and molecular outcome parameters (including serial CSF assessments) in a 29-year-old MNGIE patient undergoing 1 year of extensive HMD. We demonstrate that HMD only transiently restores increased serum and urine levels of thymidine and deoxyuridine, but fails to reduce CSF levels of the toxic metabolites and is ineffective to influence neurological function. These findings have direct important implications for clinical practice: They prevent a burdensome, long-term invasive, but ultimately probably ineffective procedure in future MNGIE patients.
\end{abstract}

Keywords: MNGIE, Haemodialysis, Mitochondriopathy, Thymidine phosphorylases

\section{Introduction}

Mitochondrial neurogastrointestinal encephalomyopathy (MNGIE) is a rare, autosomal-recessive mitochondrial disorder caused by mutations in TYMP encoding for the thymidine phosphorylase [1,2]. Clinically, MNGIE comprises a multisystemic syndrome of progressive leukoencephalopathy, ophthalmoparesis, demyelinating peripheral neuropathy, cachexia and gastrointestinal dysmotility $[1,2]$. Biallelic carriers of TYMP mutations show reduced thymidine phosphorylase enzyme activity resulting in elevation of thymidine and deoxyuridine. Accumulation of these toxic metabolites

\footnotetext{
* Correspondence: benjamin.roeben@med.uni-tuebingen.de

${ }^{\dagger}$ Equal contributors

${ }^{1}$ Department of Neurodegeneration, Hertie Institute for Clinical Brain Research $(\mathrm{HIH})$, University of Tübingen, 72076 Tübingen, Germany

${ }^{2}$ German Center for Neurodegenerative Diseases (DZNE), 72076 Tübingen, Germany

Full list of author information is available at the end of the article
}

leads to unbalanced intramitochondrial deoxynucleotide pools, which in turn cause site-specific mitochondrial DNA (mtDNA) depletion, multiple deletions and point mutations $[1,3,4]$. Several recent studies have demonstrated that it is indeed this nucleoside accumulation, rather than the deficiency of thymidine phosphorylase per se, that accounts for the molecular and phenotypic alterations in MNGIE [4].

Based on this pathophysiologic rationale, nucleoside reduction by dialysis has been proposed as a promising therapy in MNGIE patients [5, 6]. However, all studies so far did not measure these metabolites in cerebrospinal fluid (CSF), which is, however, the crucial compartment for CNS damage in MNGIE. Nor has any treatment study been conducted prospectively or with predefined quantitative outcome measures so far.

We here report a prospective, longitudinal case study with serial testing of predefined clinical and nucleoside 


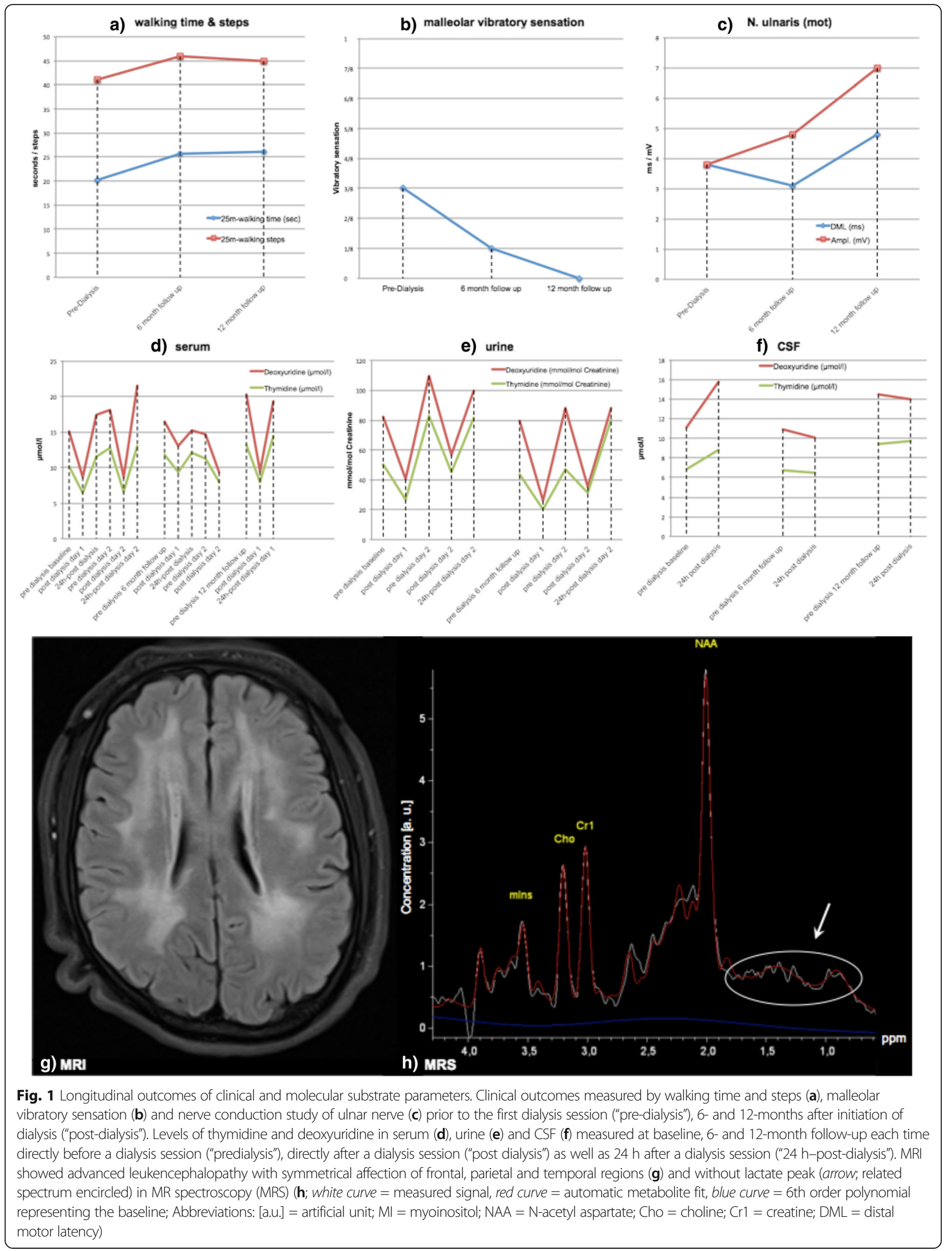


outcome parameters (including serial CSF assessments) of a male MNGIE patient undergoing 1 year of extensive hemodialysis (HMD).

\section{Case report}

A 29-year-old male of Turkish decent presented with progressive gait ataxia, external ophthalmoplegia, ptosis, sensorineural hearing loss (meanwhile supplied with cochlear implants), cachexia (body weight/height/BMI before HMD: $55 \mathrm{~kg} / 1.55 \mathrm{~m} / 22.9 \mathrm{~kg} / \mathrm{m}^{2}$; after HMD: $50 \mathrm{~kg} / 1.55 \mathrm{~m} / 20.8 \mathrm{~kg} / \mathrm{m}^{2}$ ), advanced leukencephalopathy (Fig. $1 \mathrm{~g}$ ) and recurrent, diarrhea since age 18 years. Clinical examination showed short stature $(149 \mathrm{~cm})$, dysmorphic auricles, congenital Pes cavus and severe demyelinating sensorimotor polyneuropathy. Serum lactate $(2.8-3.1 \mathrm{mmol} / \mathrm{l}$; reference range: $0.5-2.2 \mathrm{mmol} / \mathrm{l})$ and CSF lactate $(3.5-4.6 \mathrm{mmol} / \mathrm{l}$; reference range: 0 $2.2 \mathrm{mmol} / \mathrm{l}$ ) were elevated. Whole-exome sequencing (WES) revealed a homozygous TYMP stop mutation (c.112G > T, p.Glu38Stop) confirming the diagnosis of MNGIE (for WES methods and family pedigree, see Additional file 1).

Effectiveness of HMD was determined by the following predefined outcome measures: $25-\mathrm{m}$ walking-time and -steps, vibratory sensation, nerve conduction studies and levels of thymidine and deoxyuridine in serum, urine and CSF. HMD was delivered for 12 months with an initial frequency of 3 times weekly, escalated to 4 times weekly after 6 months (for details of clinical and molecular outcome assessments and HMD parameters, see Additional file 1).

After 12-month of HMD, all clinical outcome parameters indicated progression of disease, demonstrated by worsening of SARA score (Scale for the assessment of Ataxia; 11 to 13 points), decline in MoCA score (Montréal Cognitive Assessment; 27/30 to 24/30 points) and nerve conduction measures (Fig. 1a-c). Corresponding to these these clinical observations of progressive worsening, also the subject himself did not perceive any deceleration of disease progression within the 12 months of HMD compared to the pre-HMD disease progression.

Serial testing of serum and urine levels of thymidine and deoxyuridine showed transient decreases each time after dialysis, demonstrating a reproducible immediate effect of HMD. However, they returned to baseline levels within $24 \mathrm{~h}$ and did not decrease after 6 and 12 months (Fig. 1d, e). CSF levels changed neither short-term (within $24 \mathrm{~h}$ ) nor long term (at months 6 and 12) (Fig. 1f).

\section{Discussion}

We present the first prospective investigation on the effectiveness of HMD in MNGIE, capturing multiple predefined outcome measures. Our results demonstrate that HMD only transiently reduces increased serum and urine levels of thymidine and deoxyuridine and is ineffective to influence clinical disease progression.

At the same time, these results provide some preliminary insights into the HMD-associated kinetics of these two metabolites in three different body fluid compartments: urine, serum and CSF. HMD was able to transiently decrease both metabolites in urine by about $50 \%$ directly after dialysis at each of the four assessment time points, showing a reproducible relative share of urinary metabolite clearance even after repeated dialysis over 12 months. However, neither the basal levels nor the maximum levels reduced over time. This urinary clearance was paralleled by a reduction of serum levels by $20-50 \%$ directly after dialysis at each of the four assessment time points (see Fig. 1d). This suggests that the kinetics of the metabolites in both compartments runs largely in parallel. Our 6-months follow-up long-term data do not provide any clear evidence for a (e.g. compensatory) increase in synthesis of metabolites in any of the two compartments (see Fig. 1d, e). Importantly, we show for the first time that HMD fails to achieve a sustained reduction of these metabolites also in the CSF compartment, which might explain the missing neurological benefit.

Our findings thus question the alleged efficacy of dialysis, which has been based so far only on retrospective case studies of peritoneal dialysis, lacking predetermined quantitative outcome measures and long-term measurement of metabolites [5, 6]. Also alternative therapeutic options in MNGIE, including liver [7] and stem cell transplantation [3], need to be critically tested by prospective studies with predefined outcome measures.

Our report has thus direct important implications for clinical practice: it prevents a burdensome, long-term invasive, but finally probably ineffective procedure in MNGIE patients.

\section{Additional file}

Additional file 1: Whole exome sequencing, Pedigree of the index patient, Clinical and molecular substrate outcome parameters and hemodialysis parameters. (PDF $106 \mathrm{~kb}$ )

\section{Abbreviations \\ CNS: Central nervous system; CSF: Cerebrospinal fluid; HMD: Hemodialysis; MNGIE: Mitochondrial neurogastrointestinal encephalomyopathy; MoCA: Montréal Cognitive Assessment; MRI: Magnetic resonance imaging; mtDNA: mitochondrial DNA; SARA: Scale for the assessment of ataxia}

\section{Acknowledgments}

We thank the participants for participating in this study. We like to acknowledge excellent analytical support of S. Behringer.

\section{Funding}

This research was supported by the Else Kröner-Fresenius-Stiftung (award to MS)). TBH work was supported by the German Federal Ministry of Education and Research (BMBF) within the framework of the e:Med research and funding concept (grant \#FKZ 01ZX1405C). 


\section{Availability of data and materials}

Please contact author for data requests.

\section{Authors' contributions}

Collection and analysis of data: BR, JM, BB, HJB, TBH, ISA, LS, HB, LS, MS Drafting of the manuscript: BR, JM, MS. Critical review of the manuscript: $B R$, $J M, B B, H J B, T B H, I S A, L S, H B, L S, M S$. All authors read and approved the final manuscript.

\section{Ethics approval and consent to participate}

The study was performed in accordance with the ethical standards laid down in the 1964 Declaration of Helsinki and its later amendments. All individuals gave written informed consent prior to their inclusion in the present study.

\section{Consent for publication}

Consent for publication was obtained from every patient/legal guardian.

\section{Competing interests}

B. R. received travel funding from Actelion Pharmaceuticals, unrelated to the current project and manuscript. B. B. received travel funding by Bayer Vital, unrelated to the current project and manuscript. M. S. received speaker's honoraria and research support from Actelion Pharmaceuticals, unrelated to the current project and manuscript. J. M., H. B., T. B. H., I. S-A., L. S. and H. J. B. have nothing to disclose.

\section{Publisher's Note}

Springer Nature remains neutral with regard to jurisdictional claims in published maps and institutional affiliations.

\section{Author details}

${ }^{1}$ Department of Neurodegeneration, Hertie Institute for Clinical Brain Research (HIH), University of Tübingen, 72076 Tübingen, Germany. ${ }^{2}$ German Center for Neurodegenerative Diseases (DZNE), 72076 Tübingen, Germany. ${ }^{3}$ Department of Epileptology, Hertie Institute for Clinical Brain Research (HIH), University of Tübingen, 72076 Tübingen, Germany. ${ }^{4}$ Department of Neuroradiology, University of Tübingen, 72076 Tübingen, Germany. ${ }^{5}$ Department of Child Nephrology, University Children's Hospital Tübingen, 72076 Tübingen, Germany. ${ }^{6}$ Institute of Human Genetics, Technische Universität München, 81675 Munich, Germany. ${ }^{7}$ Institute of Human Genetics, Helmholtz Zentrum München, 85764 Neuherberg, Germany. ${ }^{8}$ Institute of Medical Genetics and Applied Genomics, University of Tübingen, 72076 Tübingen, Germany. ${ }^{9}$ Department of Pediatric Neurology and Developmental Medicine, University Children's Hospital Tübingen, 72076 Tübingen, Germany. ${ }^{10}$ Laboratory of Clinical Biochemistry and Metabolism, Department of General Pediatrics, Adolescent Medicine and Neonatology, University Medical Centre Freiburg, Freiburg, Germany.

Received: 17 March 2017 Accepted: 25 July 2017

Published online: 01 August 2017

\section{References}

1. Hirano M, Silvestri G, Blake DM, Lombes A, Minetti C, Bonilla E, Hays AP, Lovelace RE, Butler I, Bertorini TE, et al. Mitochondrial neurogastrointestinal encephalomyopathy (MNGIE): clinical, biochemical, and genetic features of an autosomal recessive mitochondrial disorder. Neurology. 1994;44(4):721-7.

2. Nishino I, Spinazzola A, Hirano M. Thymidine phosphorylase gene mutations in MNGIE, a human mitochondrial disorder. Science. 1999:283(5402):689-92.

3. Halter JP, Michael W, Schupbach M, Mandel H, Casali C, Orchard K, Collin M, Valcarcel D, Rovelli A, Filosto M, et al. Allogeneic haematopoietic stem cell transplantation for mitochondrial neurogastrointestinal encephalomyopathy. Brain. 2015;138(Pt 10):2847-58.

4. Yadak R, Sillevis Smitt P, van Gisbergen MW, van Til NP and de Coo IFM. Mitochondrial Neurogastrointestinal Encephalomyopathy Caused by Thymidine Phosphorylase Enzyme Deficiency: From Pathogenesis to Emerging Therapeutic Options. Front. Cell. Neurosci. 2017;11:31. doi:10.3389/ fncel.2017.00031.

5. Yavuz H, Ozel A, Christensen M, Christensen E, Schwartz M, Elmaci M, Vissing J. Treatment of mitochondrial neurogastrointestinal encephalomyopathy with dialysis. Arch Neurol. 2007;64(3):435-8.
6. Sivadasan A, Muthusamy K, Patil AK, Mathew V, Alexander M. Pearls \& OySters: mitochondrial neurogastrointestinal encephalomyopathy: diagnosis and response to peritoneal dialysis. Neurology. 2016;86(14):e147-50.

7. De Giorgio R, Pironi L, Rinaldi R, Boschetti E, Caporali L, Capristo M, Casali C, Cenacchi G, Contin M, D'Angelo R, et al. Liver transplantation for mitochondrial neurogastrointestinal encephalomyopathy. Ann Neurol. 2016;80(3):448-55.

\section{Submit your next manuscript to BioMed Central and we will help you at every step:}

- We accept pre-submission inquiries

- Our selector tool helps you to find the most relevant journal

- We provide round the clock customer support

- Convenient online submission

- Thorough peer review

- Inclusion in PubMed and all major indexing services

- Maximum visibility for your research

Submit your manuscript at www.biomedcentral.com/submit
Biomed Central 